\begin{tabular}{|c|c|}
\hline & $\begin{array}{l}\text { International Journal of Trend in Scientific } \\
\text { Research and Development (IJTSRD) }\end{array}$ \\
\hline 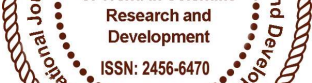 & International Open Access Journal \\
\hline 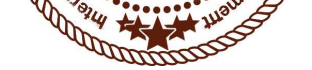 & ISSN No: 2456 - 6470 | www.ijtsrd.com | Volume - 2 | Issue - 2 \\
\hline
\end{tabular}

\title{
An Ethnobotanical Survey of Medicinal, Ornamental, Agricultural and Wild Plants in Maruthuvambadi Village, Tiruvannamalai District, Tamilnadu, India
}

Vijayaraj, $R$

Ph.D. Research Scholar, Department of Plant

Biology and Biotechnology \& Loyola

Institute of Frontier Energy (LIFE),

Loyola College, Nungambakkam,

Chennai, Tamil Nadu
Jaquline Chinna Rani, I.

Assistant Professor, Department of Plant

Biology and Biotechnology \& Loyola

Institute of Frontier Energy (LIFE),

Loyola College, Nungambakkam,

Chennai, Tamil Nadu

\section{ABSTRACT}

Ethnobotanical survey and documentation of medicinal, field grown, ornamental, and wild plants were carried out in and around 'Maruthuvambadi' village, Tiruvannamalai district, Tamilnadu. This study aimed to identify plants collected for medicinal and other purposes by the local people of the village. A total of 250 plant species were observed in this study. These plant species, belonging to 70 families with 199 genuses are listed in alphabetical order with family and vernacular name. As a result of global warming, pollution and deforestation some precious plants are coming under endangered list so the information obtained from this simple survey will be beneficial to the upcoming generations of this village in identifying the medicinally valuable plants.

Keywords: Commodities; Crotons; Herbals; Lakes; Rice; Rocks

\section{Introduction}

Maruthuvambadi village is in Tiruvannamalai district, Tamil Nadu state, India. The village is located at end of Sorakolathur forest and in between Vellore highways. Tiruvannamalai is the nearest town to Maruthuvambadi which is approximately $20 \mathrm{~km}$ away toward North. One hundred seventy-one kilometers away from the capital of Tamil Nadu that is Chennai.
The junction which connects the village to main road is called Kondam; this is the highway of Vellore. Kariyandal $(1 \mathrm{~km})$, Sorakolathur $(2 \mathrm{~km})$, Naidumangalam $(4 \mathrm{~km})$ and Vadapuzhudiyur $(4 \mathrm{~km})$ are the nearby Villages to Maruthuvambadi. Sorakolathur reserve forest (Fig. 1) is a source of many fauna and flora. This forest belongs to Sorakolathur village panchayath. According to Census 2011 information (Census, 2011) the location code or village code of Maruthuvambadi is 632075 . The total geographical area of village is 397.Eleven hectares and the village has a total population of 2,090 (Male1,039 and Female-1,051). There are about 499 families in Maruthuvambadi village. Tamil is the native language. In Tamil language 'Maruthuvam' means related to medicine, some aged men and women told that this village is filled with different types of medicinal plants therefore this name came. Tiruvannamalai is industrially backward district in Tamil Nadu state (Ministry of MSME, 2012-2013). Javadhu hills with many outcrops were situated in the borders of both Tiruvannamalai and Vellore districts (Poongani, 2017). Agriculture is the major occupation. Paddy, groundnut and jaggery are the three most important commodities (Chart.1) which are reaped and exported to town from this village. 


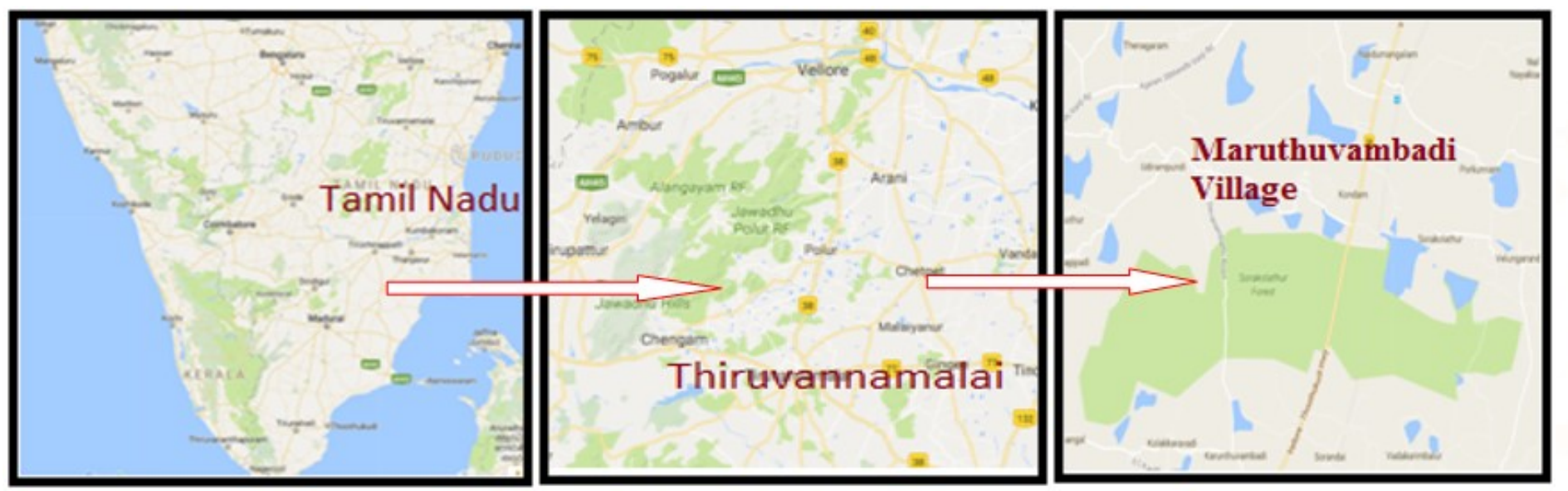

Fig 1: Road map toward Maruthuvambadi Village, Tiruvannamalai District, Tamilnadu, India (Vellore District Highway)

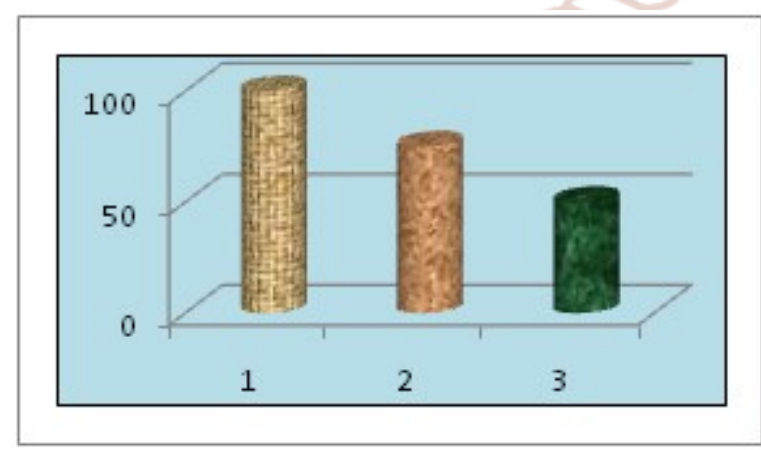

Chart 1: Top three commodities imported to town.

(1) Rice from Oryza sativa (2) Groundnut from Arachis hypogeal (3) Jaggery from Saccharum officinarum

\section{Materials and Methods}

\section{Ethnobotanical Study}

There is very limited information available regarding medicinal plants used by traditional healers and general people in villages, for treating common ailments and diseases. It is very urgent need for identifying and documenting these valuable resources before they become inaccessible and extinct. Ethnobotanical survey is highly needed for the conservation of plants and represents the preliminary information required for future phyto-chemical investigation. Medicinal plants are accessible and cheap so $80 \%$ of people in developing countries used these for treating many health problems (Anup, 2014). The use of plants and plant products for different purposes such as food, fodder, medicine, fiber, etc., could be traced as far back as to the beginning of human civilization (Bhattarai, 2016). The indigenous system of medicine namely Ayurvedic, Siddha and Unani have been in existence for several centuries.
This system of medicine helps the needs of nearly seventy percent of our population residing in the villages. The phytoconstituents of these plants were responsible for healing diseases (Disticraj, 2015). The study of bioactivity of these plants could lead to the discovery of novel broad spectrum natural products (Gbadamosi, 2014). The most common way of preparing remedies from herbs is decoctions, by boiling plant parts in a large amount of water until this is considerably reduced and colored by plants phytochemicals. In some of the decoctions sugarcane is added to prepare medicinal syrups (Giovannini, 2015). Among the different plant parts used for the preparation of medicine, the leaves were found to be the most frequently used plant parts in the preparation of remedies (Ignacimuthu, 2008). Ethnobotany is the study of the interactions and relationships between plants and people over time and space. This includes the uses, knowledge, beliefs, management systems, classification systems and language that both modern and traditional cultures have for plants and their associated terrestrial and aquatic ecosystems (Johnson, 2015). Oldest people always have enormous knowledge about medicinal uses of plants and this knowledge is mostly undocumented and transmitted orally from generation to generation. Therefore, it is highly needed to explore and document this unique and indigenous, traditional knowledge of the senior citizens, before it diminishes (Lingaiah, 2013). The fact that such high number of species is used to treat many different medical problems and have so many therapeutic applications (Manuel, 2005). The various plant parts used included whole plant, leaf, root, stem, flower, seed, bark, gum, fruit, petiole, and rhizome (Mohammed, 2009). Ethno medicines are developed by the ethnobotanical and ethnopharmacological 
surveys. These herbal medicines have less side effects and man can get the herbs easily from nature (Omwenga, 2014). Ethnobotanical study is of immense importance with medical science. Now it is being as a well established branch of science with much attention (Pavun Kumar, 2011). Medicinal plant lore or herbal medicine is a major component of traditional medicine (Ramakrishnan). In general, the traditional medicine treats patients only in the morning times (Savithramma, 2007). In this world, some countries are gifted with the wealth of medicinally important plants (Shah, 2013). In the last few decades there has been an exponential growth in the field of herbal medicine (Thamacin, 2014). The biological resources are indispensable for economic growth and development of any nation (Vijayasankar, 2012).

\section{Study Area}

The study area selected for the ethnobotanical survey of different plant varieties was carried out in Maruthuvambadi village and Sorakolathur reserve (Fig. 2) forest. We can see the dome of Tiruvannamalai hill from this village. Two different colors in single flower called 'vedethalam' are also available here. Paddy and sugarcane field gives additional greenish to the village. A biggest rare tree Basia latifolia-(Iluppaimaram) is standing (Fig. 4) here like a huge giant, monkeys use to play on its branches and they can eat tasty fruits of this tree and seeds are used to make soap oil. Many rocks are there

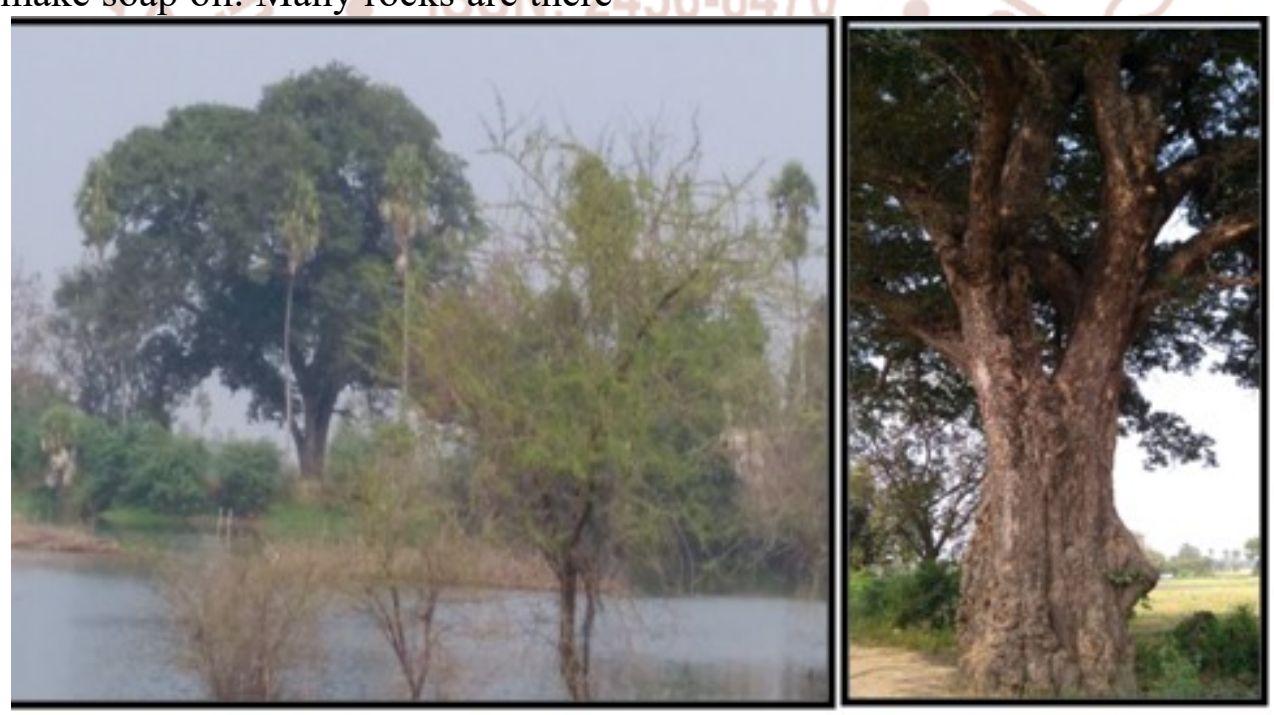

Fig 4: Biggest and Oldest tree of the village (Basia latifolia-Iluppaimaram)

\section{Result and Discussion}

This is the first report of plants of Maruthuvambadi village. A field trip was conducted few times and the in the forest and five different lakes (Fig. 3) are surrounded in this village as water resources for agriculture.

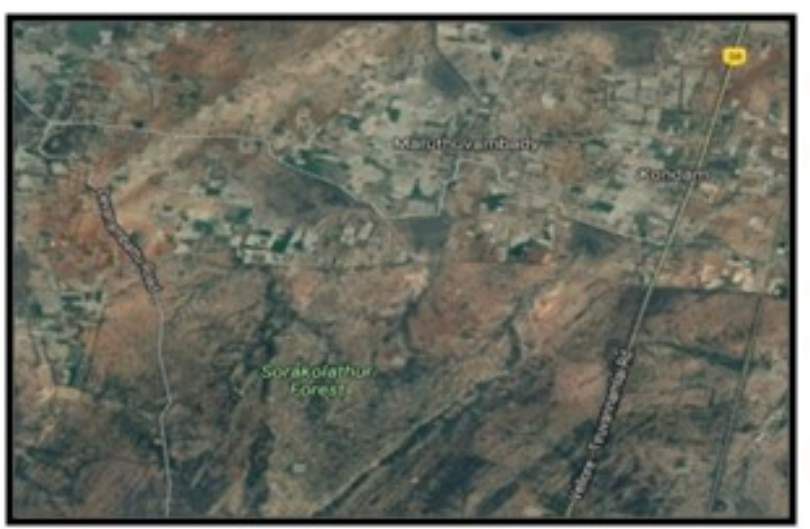

Fig 2: Study Area Sorakolathur Forest.

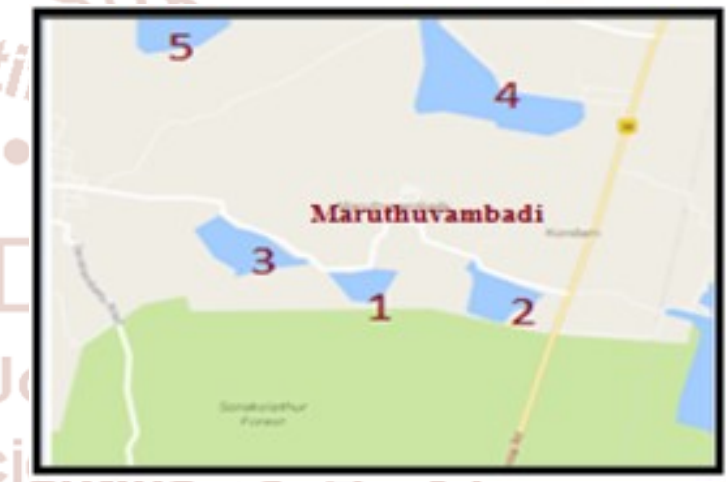

Fig 3: Google map shows five different lakes around the village (1) Chitheri (2) Kariyandaleri (3) Periyeri (4) Puzhuthiureri (5) Vennapanthaleri. 
collective number of plants observed during the field work was199 genus, 250 species which were distributed among 70 families. Acanthaceae-(8), Aizoaceae-(1), Amaranthaceae-(7), Amaryllidaceae(1), Anacardiaceae-(2), Annonaceae-(2), Apiaceae(2), Apocynaceae-(11), Araceae-(2), Arecaceae-(4), Aristolochiaceae-(1),

Asclepiadaceae(5), Asparagaceae-(5), Asphodelaceae-(1), Asteraceae(7), Balsaminaceae-(1), Basellaceae-(2), Brassicaceae-(6), Burseraceae-(1), Cactaceae-(1), Caesalpinaceae-(1), Capparaceae-(1), Caricaceae-(1), Cleomaceae-(2), Colchicaceae-(1), Commelinaceae(1), Convolvulaceae-(6), Cucurbitaceae-(13), Cyperaceae-(1), Ebenaceae-(1), Euphorbiaceae-(12), Fabaceae-(40), Gisekiaceae-(1), Lamiaceae-(4), Lauraceae-(1), Leguminaceae-(1), Liliaceae-(2), Lythraceae-(3), Malvaceae-(10), Marsileaceae-(1), Meliaceae-(2), Menispermaceae-(1), Moraceae-(3), Moringaceae-(1), Musaceae-(1), Myrtaceae-(3), Nyctaginaceae-(3), Nymphaeaceae-(1), Oleaceae-(1), Oxalidaceae-(1), Papaveraceae-(1), Passifloraceae(1), Pedaliaceae-(1), Phyllanthaceae-(3), Poaceae(12), Portulacaceae-(3), Rhamnaceae-(2), Rosaceae(2), Rubiaceae-(5), Rutaceae-(8), Salicaceae-(1),
Salvadoraceae-(1), Sapindaceae-(2), Sapotaceae-(2), Smilacaceae-(1), Solanaceae-(9), Verbenaceae-(2), Violaceae-(1), Vitaceae-(2), Zygophyllaceae-(1). More number of observed species comes under the families Fabaceae-(40), Cucurbitaceae-(13), Euphorbiaceae-(12), Poaceae-(12), Apocynaceae-(11) and Malvaceae-(10).

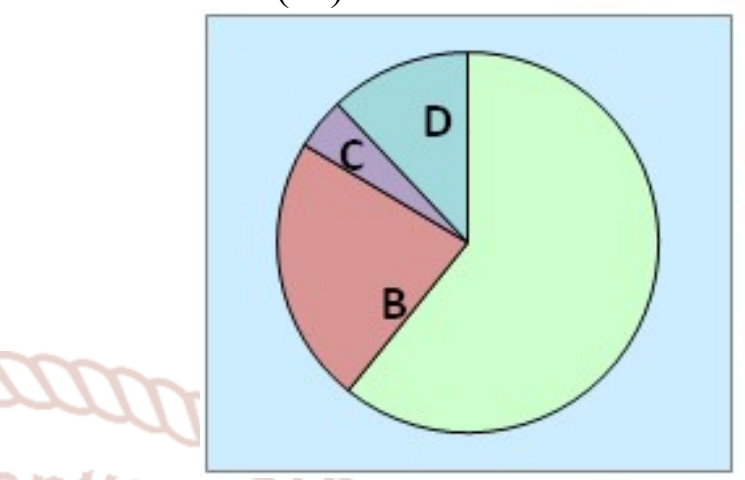

Chart 2: Percentage of plant varieties in Maruthuvambadi Village

(A) Medicinal plants (B) Agricultural

(C) Ornamentals (D) Wild plants

\section{Data analysis}

From the data collected, we concluded that this village is highly filled with (Chart.2) medicinal plants. Different species were listed in alphabetical order by scientific, family and local name (Table-1, 2,3 and 4).

Table 1: List of medicinal plants available in Maruthuvambadi village

\begin{tabular}{|l|l|l|l|}
\hline S.N & Botanical name & Family & Vernacular name \\
\hline 1 & Abrus precatorius L. & Fabaceae & Gundumani \\
\hline 2 & Abutilon indicum (Link) Sweet. & Malvaceae & Thuthi \\
\hline 3 & Acalypha indica L. & Euphorbiaceae & Kuppaimeni \\
\hline 4 & Achyranthes aspera L. & Amaranthaceae & Naaiuruvi \\
\hline 5 & Adananthera pavoniana L. & Fabaceae & Aanaigundumani \\
\hline 6 & Adhatoda vasica (L.) Nees. & Acanthaceae & Adathodai \\
\hline 7 & Aegle marmalos (L.) Corrêa. & Rutaceae & Vilvam \\
\hline 8 & Aerva lanata L.) Juss. ex Schult. & Amaranthaceae & Sirukanpeelai \\
\hline 9 & Aeschynomene aspera L. & Fabaceae & Kidaichi, Netti \\
\hline 10 & Agave americana L. & Asparagaceae & Aanaikatrazhai \\
\hline 11 & Aloe vera (L.) Burm.f. & Asphodelaceae & Sotrukkatrazhai \\
\hline 12 & Alternanthera sessilis (L.) R.Br. ex DC. & Amaranthaceae & Ponnaankanni \\
\hline 13 & Amaranthus spinosa L. & Amaranthaceae & Mullu keerai \\
\hline 14 & Ammannia baccifera L. & Lythraceae & Neermeal neruppu \\
\hline & $\begin{array}{l}\text { Andrographis } \\
\text { (Burm.f.) Wall. ex Nees. }\end{array}$ paniculata & Acanthaceae & Nila vembu, Siriyaa nangai \\
\hline 16 & Annona squamosa L. & Annonaceae & Seetha pazham \\
\hline 17 & Argemone mexicana L. & Papaveraceae & Kudiyotti \\
\hline
\end{tabular}


International Journal of Trend in Scientific Research and Development (IJTSRD) ISSN: 2456-6470

\begin{tabular}{|c|c|c|c|}
\hline 18 & Aristolochia bracteolata Lam. & Aristolochiaceae & Aaduthinnapalai, Eeswaramooli \\
\hline 19 & Asystasia gangetica (L.) T.Anderson. & Acanthaceae & Mithikeerai, Pattaasukaai \\
\hline 20 & Azadirachta indica A. Juss. & Meliaceae & Vembu \\
\hline 21 & Barleria cristata L. & Acanthaceae & Neela December, Vellai December \\
\hline 22 & Barleria prionitis L. & Acanthaceae & Manjal December \\
\hline 23 & Boerhaavia diffusa L. nom. cons. & Nyctaginaceae & Mookuratai keerai \\
\hline 24 & Borassus flabellifer L. & Arecaceae & Panaimaram \\
\hline 25 & Borreria hispida (L.) K. Schum. & Rubiaceae & Nathai choori \\
\hline 26 & Broyonia dioica Jacq. & Cucurbitaceae & Naaipaagarkaai \\
\hline 27 & Caesalpinia bonduc (L.) Roxb. & Caesalpinaceae & Kalarchi kodi, Kokkimullusedi \\
\hline 28 & Calotropis gigantea (L.) W.T.Aiton. & Apocynaceae & Erukku \\
\hline 29 & Calotropis procera (Aiton) W.T.Aiton. & Asclepiadaceae & Velerukku \\
\hline 30 & Cammelina benghalensis L. & Commelinaceae & $\begin{array}{l}\text { Kozhikannankeerai, } \\
\text { vaazhai }\end{array}$ \\
\hline 31 & Canavalia ensiformis (L.) DC. & Fabaceae & Thambattankaai \\
\hline 32 & Caralluma fimbriata Wall. & Asclepiadaceae & Kallimudayan \\
\hline 33 & Caralluma umbellata Roxb. & Asclepiadaceae & Aanai kallimudayan \\
\hline 34 & Cardiospermum halicacabum L. & Sapindaceae & Mudakkathan \\
\hline 35 & Carica papaya L. & Caricaceae & Pappali \\
\hline 36 & Carissa carandas L. & Apocynaceae & Kaattukala, Sirukala \\
\hline 37 & Carissa spinarum L. & Apocynaceae & Kalakaasedi \\
\hline 38 & Cassia tora $\mathrm{L}$. & Caesulpinaceae & Thagarai \\
\hline 39 & Cassia nodosa Roxb. & Fabaceae & Kungilium \\
\hline 40 & Cassia obtusifolia L. & Fabaceae & Oosithagarai \\
\hline 41 & Cassia siamea Lam. & Fabaceae & Seemaiagathi \\
\hline 42 & Cassytha filiformis L. & Lauraceae & Koothan kodi, Pulluruvi \\
\hline 43 & Catharanthus roseus (L.) G.Don. & Apocynaceae & Nithyakalyaani \\
\hline 44 & Ceiba pentandra (L.) Gaertn. & Malvaceae & Ilavampanju \\
\hline 45 & Cissus quadrangularis L. & Vitaceae & Pirandai \\
\hline 46 & Cissus repens Lam. & Vitaceae & Sempirandai \\
\hline 47 & Citrus aurantifolia (Christm.) Swingle. & Rutaceae & Kaderankaai \\
\hline 48 & Citrus aurantium L. & Rutaceae & Kolanjika, Kichili \\
\hline 49 & Citrus limetta Risso. & Rutaceae & Sathukudi \\
\hline 50 & Citrus medica L. & Rutaceae & Elumichai \\
\hline 51 & Cleome gynandra L. & Cleomaceae & Kaattukadugu \\
\hline 52 & Cleome viscosa L. & Cleomaceae & Naaikadugu \\
\hline 53 & Clitoria ternatea L. & Fabaceae & Sangu poo \\
\hline 54 & Coccinia grandis (L.) Voigt. & Cucurbitaceae & Kasappukovaikaai \\
\hline 55 & Cocos nucifera L. & Arecaceae & Thennai \\
\hline 56 & Commiphora caudata (Wight \& Arn.) & Burseraceae & Kiluvai \\
\hline 57 & Cordia dichotoma G.Forst. & Boraginaceae & Mookuchalhi pazham \\
\hline 58 & Cucumis melo var. agrestis & Cucurbitaceae & Sukkankaai \\
\hline 59 & Cuscuta reflexa Roxb. & Convolvulaceae & Ammaiyar koonthal \\
\hline 60 & $\begin{array}{l}\text { Cymbopogon goeringii } \quad \text { (Steud.) } \\
\text { A.Camus. }\end{array}$ & Poaceae & Ootanguchi, Manji \\
\hline 61 & Cynodon dactylon (L.) Pers. & Poaceae & Arugampul \\
\hline 62 & Cyperus rotundus L. & Cyperaceae & Korai \\
\hline 63 & Datura metel L. & Solanaceae & Oomatham \\
\hline 64 & Delonix regia Raf. & Fabaceae & Poomaram, Vaathanarayan \\
\hline 65 & Diplocyclos palmatus (L.) C.Jeffrey. & Cucurbitaceae & Aiviralkkovai \\
\hline
\end{tabular}


International Journal of Trend in Scientific Research and Development (IJTSRD) ISSN: 2456-6470

\begin{tabular}{|c|c|c|c|}
\hline 66 & Dodonaea viscosa Jacq. & Sapindaceae & Viralipoo \\
\hline 67 & Eclipta prostrata L. & Asteraceae & Karisalankanni \\
\hline 68 & Erythrina stricta Roxb. & Fabaceae & Murukku maram \\
\hline 69 & Eucalyptus globulus Labill. & Myrtaceae & Thailamaram, Neelagiri \\
\hline 70 & Euphorbia antiquorum L. & Euphorbiaceae & Sathurakalli \\
\hline 71 & Euphorbia hetrophylla L. & Euphorbiaceae & Paalperukki \\
\hline 72 & Euphorbia hirta L. & Euphorbiaceae & Ammanpacharisi \\
\hline 73 & Euphorbia neriifolia L. & Euphorbiaceae & Ilaikalli \\
\hline 74 & Euphorbia tirucalli L. & Euphorbiaceae & Kalli \\
\hline 75 & Evolvolus alsinodes L. & Convolvulaceae & Vishnukiranthi \\
\hline 76 & Ficus benghalensis L. & Moraceae & Aalamaram \\
\hline 77 & Ficus religiosa L. & Moraceae & Arasamaram \\
\hline 78 & Flacourtia indica (Burm. f.) Merr. & Salicaceae & Sothaikalapazham \\
\hline 79 & Gloriosa superba L. & Colchicaceae & Senkaanthal, Kanvalipoo \\
\hline 80 & Heliotropium indicum L. & Boraginaceae & Thelkodukku \\
\hline 81 & Hemidesmus indicus (L.) R.Br. & Apocynaceae & Nannaari \\
\hline 82 & Hibiscus rosa sinensis L. & Malvaceae & Semparuthi \\
\hline 83 & Hybanthus enneaspermus (L.) F.Muell. & Violaceae & Orithazhthaamarai \\
\hline 84 & Hygrophylia auriculata Schumach. & Acanthaceae & Neermulli \\
\hline 85 & Indigofera aspalathoides Vahl. & Fabaceae & Sivanarvembu \\
\hline 86 & Ipomoea hederacea Jacq. & Convolvulaceae & Kodikaathan \\
\hline 87 & Ipomoea obscura (L.) Ker Gawl. & Convolvulaceae & Siruoonam, Siruthalai \\
\hline 88 & Jatropha gossypifolia L. & Euphorbiaceae & Kattuaamanakku \\
\hline 89 & Justicia adhatoda L. & Acanthaceae & Adathoda \\
\hline 90 & Lawsonia inermis L. & Lythraceae & Maruthaani, Azhavanathazhai \\
\hline 91 & Leucas aspera (Willd.) Link. & Lamiaceae & Thumbai \\
\hline 92 & Leucas indica (L.) R.Br. ex Vatke. & Lamiaceae & Kavizh thumbai \\
\hline 93 & Limonia acidissima $\mathrm{L}$ & Rutaceae & Vilaam pazham \\
\hline 94 & Lippia nodiflora L. & Verbenaceae & Poduthalai \\
\hline 95 & Mangifera indica L. & Anacardiaceae & Maamaram \\
\hline 96 & Melia azedarach L. & Meliaceae & Malai veambu \\
\hline 97 & Mimosa amara Roxb. & Fabaceae & Oosilmaram \\
\hline 98 & Morinda tinctoria Roxb. & Rubiaceae & Nunaa \\
\hline 99 & Nymphaea lotus L. & Nymphaeaceae & Allipoo \\
\hline 100 & Ocimum basilicum L. & Lamiaceae & Karanthai, Kanjaankorai \\
\hline 101 & Ocimum sanctum L. & Lamiaceae & Thulasi \\
\hline 102 & Opuntia stricta Haw. & Cactaceae & Sappathikalli \\
\hline 103 & Oxalis corniculata L. & Oxalidaceae & Puliaarai \\
\hline 104 & Oxystelma esculantum (L.f.) Sm. & Apocynaceae & Oosipaalai \\
\hline 105 & Parthenium hysterophorus L. & Asteraceae & Kenathuppoondu \\
\hline 106 & Passiflora foetida L. & Passifloraceae & Kurangupazham \\
\hline 107 & Pergularia daemia (Forssk.) Chiov. & Asclepiadaceae & Uthaamani, Uthamakannigai \\
\hline 108 & Phyllanthus acidus (L.) Skeels. & Phyllanthaceae & Arainellikaai \\
\hline 109 & Phyllanthus emblica L. & Phyllanthaceae & Kaatunelli \\
\hline 110 & Phyllanthus niruri L. & Phyllanthaceae & Keezhanelli \\
\hline 111 & Phyllanthus reticulatus Poir. & Euphorbiaceae & Pallukuchithazhai \\
\hline 112 & Physalis minima L. & Solanaceae & Tharmathakkali \\
\hline 113 & Psidium guajava L. & Myrtaceae & Koiya \\
\hline 114 & Pithecellobium dulce (Roxb.) Benth. & Fabaceae & Kodukapuli, Sulaikaai \\
\hline 115 & Plumeria rubra L. & Apocynaceae & Paneerpoo, Kaathuvalipoo \\
\hline
\end{tabular}


International Journal of Trend in Scientific Research and Development (IJTSRD) ISSN: 2456-6470

\begin{tabular}{|c|c|c|c|}
\hline 116 & Pongamia pinnata (L.) Pierre. & Fabaceae & Pungamaram \\
\hline 117 & Prosopis juliflora (Sw.) DC. & Fabaceae & Vealikaathaan \\
\hline 118 & Punica granatum L. & Lythraceae & Maadulai \\
\hline 119 & Randia dumatorum Lam. & Rubiaceae & Aathalankaai \\
\hline 120 & Ricinus communis L. & Euphorbiaceae & Aamanakku \\
\hline 121 & Saccharum spotaneum L. & Poaceae & Naanal \\
\hline 122 & Samanea saman F.Muell. & Fabaceae & Thoongu moonji maram \\
\hline 123 & Sansevieria roxburghiana Sch. & Asparagaceae & Marul \\
\hline 124 & Sansevieria trifasciata Prain. & Asparagaceae & Kaatumarul \\
\hline 125 & Sarcostemma intermedium Decne. & Asclepiadaceae & Kodi kalli \\
\hline 126 & Securinega leucopyrus Willd. & Euphorbiaceae & Kaatupila, Pacharisipazham \\
\hline 127 & Senna auriculata (L.) Roxb. & Fabaceae & Aavarampoo \\
\hline 128 & Sida acuta Burm.f. & Malvaceae & Aruvaamanaipoondu \\
\hline 129 & Sida cordifolia L. & Malvaceae & Nilathuthi \\
\hline 130 & Smilax zeylanica L. & Smilacaceae & Kaatukodi \\
\hline 131 & Solanum trilobatum L. & Solanaceae & Thoothuvalai \\
\hline 132 & Solanum xanthocarpum Schrad. & Solanaceae & Kandankathiri \\
\hline 133 & Syzygium cumini L. & Myrtaceae & Naaval pazham \\
\hline 134 & $\begin{array}{l}\text { Tabernaemontana divaricata } \\
\text { R.Br. ex Roem. \& Schult. }\end{array}$ & Apocynaceae & Nanthiyavattam \\
\hline 135 & Tamarindus indica $\mathrm{L}$. & Fabaceae & Puliyamaram \\
\hline 136 & Tectona grandis L. & Liliaceae & Thekkumaram \\
\hline 137 & Tephrosia purpuraea L. & Fabaceae & Kollukaivelai \\
\hline 138 & Tephrosia spinosa (L.) Pers. & Fabaceae & Mugavellai \\
\hline 139 & Thespesia populnea (L.) Sol. & Malvaceae & Poovarasu \\
\hline 140 & Thevetia peruviana (Pers.) K. Schum. & Apocynaceae & Ponnarali \\
\hline 141 & Tinospora cordifolia (Thunb.) Miers. & Menispermaceae & Seenthilkodi \\
\hline 142 & Tragia ramosa Torr. & Euphorbiaceae & Poonaikasar \\
\hline 143 & Trianthema portulacastrum L. & Aizoaceae & Kuppaikeerai \\
\hline 144 & Tribulus lanuginosus L. & Zygophyllaceae & Nerunjil \\
\hline 145 & Trichodesma indicus L. & Boraginaceae & Kavizhthumbai \\
\hline 146 & Tridax procumbens L. & Asteraceae & Mookuthipoo \\
\hline 147 & Unginea indica (Roxb.) Kunt. & Liliaceae & Kaatuvengayam \\
\hline 148 & Vernonia cinerea (L.) Less. & Asteraceae & Neichati \\
\hline 149 & Wrightia tinctoria (Roxb.) R.Br. & Apocynaceae & Vetpaalai \\
\hline 150 & Xanthium strumarium L. & Asteraceae & Seepukaai \\
\hline 151 & Ziziphus jujuba Mill. & Rhamnaceae & Ilanthaimaram \\
\hline 152 & Ziziphus oenoplia (L.) Mill. & Rhamnaceae & Sooraimullu \\
\hline
\end{tabular}

Table-2. List of crops used for cultivation by farmers in Maruthuvambadi village.

\begin{tabular}{|c|c|c|c|}
\hline .S.N & Botanical name & Family & Vernacular name \\
\hline 1 & Abelmoschus esculentus (L.) Moench. & Malvaceae & Vendaikaai \\
\hline 2 & Allium cepa L. & Amaryllidaceae & Vengayam \\
\hline 3 & Arachis hypogea L. & Fabaceae & Nilakadalai, Verkadalai, Kadalaikaai \\
\hline 4 & Arum colocasia L. & Araceae & Seppangizhangu \\
\hline 5 & Basella alba L. & Basellaceae & Pasalai keerai \\
\hline 6 & Basella rubra L. & Basellaceae & Kodi pasalai, Sivappu pasalai \\
\hline 7 & Benincasa hispida (Thunb.) Cogn. & Cucurbitaceae & Kalyana poosani \\
\hline 8 & Brassica juncea L. & Brassicaceae & Kadugu \\
\hline
\end{tabular}


International Journal of Trend in Scientific Research and Development (IJTSRD) ISSN: 2456-6470

\begin{tabular}{|c|c|c|c|}
\hline 9 & Cajanus indicus. Spreng. & Fabaceae & Thuvarai \\
\hline 10 & Capsicum annum L. & Solanaceae & Milakaai \\
\hline 11 & Capsicum frutescens $\mathrm{L}$. & Solanaceae & Gundumilagaai \\
\hline 12 & Celosia argentea $\mathrm{L}$. & Amaranthaceae & Pannaikeerai \\
\hline 13 & Celosia cristata L. & Amaranthaceae & Kozhikondai poo \\
\hline 14 & Centella asiatica (L.) Urban. & Apiaceae & Vallaraikeerai \\
\hline 15 & Cephalandra indica Naud. & Cucurbitaceae & Kovaikaai \\
\hline 16 & Chrysanthemum coronarium L. & Asteraceae & Saamanthi poo \\
\hline 17 & Citrullus lanatus Thunb. & Cucurbitaceae & Tharpoosani \\
\hline 18 & Coriandrum sativam $\mathrm{L}$. & Apiaceae & Kothamalli \\
\hline 19 & Crossandra infundibuliformis (L.) Nees. & Acanthaceae & Kanakambaram \\
\hline 20 & Cucumis sativus L. & Cucurbitaceae & Vellari pazham \\
\hline 21 & Curcubita pepo L. & Cucurbitaceae & Poosani \\
\hline 22 & Dolichos biflorus L. & Fabaceae & Kollu \\
\hline 23 & Eleusine cororana Gaertn. & Gramineae & Kezhvaragu \\
\hline 24 & Gisekia pharnaceoides L. & Gisekiaceae & Manalkeerai \\
\hline 25 & Gomphrena globosa L. & Amaranthaceae & Vaadamalli \\
\hline 26 & Helianthus annuus L. & Asteraceae & Sooriyagaanthi \\
\hline 27 & Hibiscus cannabinus L. & Malvaceae & Pulichai keerai \\
\hline 28 & Hibiscus surattensis L. & Malvaceae & Kaatupulichai keerai \\
\hline 29 & Jasminum sambac (L.) Aiton. & Oleaceae & Malligai \\
\hline 30 & Lablab purpureus L. & Fabaceae & Avarai \\
\hline 31 & Lagenaria siceraria (Molina) Standl. & Cucurbitaceae & Suraikaai \\
\hline 32 & Luffa acutangula (L.) Roxb. & Cucurbitaceae & Peerkankaai \\
\hline 33 & Manihot esculenta Crantz. & Euphorbiaceae & Maravalli \\
\hline 34 & Marsilea quadrifolia L. & Marsileaceae & Aaraikeerai \\
\hline 35 & Megathyrsus maximus Jacq. & Poaceae & Theevanappul \\
\hline 36 & Momordica charantia L. & Cucurbitaceae & Paagarkaai \\
\hline 37 & Moringa oleifera Lam. & Moringaceae & Murungai \\
\hline 38 & Murraya koenigii (L.) Sprengel. & Rutaceae & Karuvepilai \\
\hline 39 & Musa paradisiaca L. & Musaceae & Vaazhai \\
\hline 40 & Nerium oleander L. & Apocynaceae & Arali \\
\hline 41 & Oryza sativa L. & Poaceae & $\mathrm{Nel}$ \\
\hline 42 & Pennisetum glaucum (L.) R.Br. & Poaceae & Kambu \\
\hline 43 & Phaseolus mungo L. & Fabaceae & Oulunthu \\
\hline 44 & Phaseolus trilobatus L. & Fabaceae & Thattaan pairu \\
\hline 45 & Polianthes tuberosa L. & Asparagaceae & Sampangipoo \\
\hline 46 & Portulaca oleracea L. & Portulacaceae & Paruppukeerai \\
\hline 47 & Raphanus sativus L. & Brassicaceae & Mullangi \\
\hline 48 & Saccharum officinarum L. & Poaceae & Karumbu \\
\hline 49 & Sesamum indicum L. & Pedaliaceae & Ellu \\
\hline 50 & Solanum melongena L. & Solanaceae & Katharikaai \\
\hline 51 & Solanum nigrum L. & Solanaceae & Manathakkali \\
\hline 52 & Solanum torvum $\mathrm{Sw}$. & Solanaceae & Sundaikaai \\
\hline 53 & Sorghum bicolor (L.) Moench & Poaceae & Solam \\
\hline 54 & Trichosanthes cucumerina L. & Cucurbitaceae & Pudalangaai \\
\hline 55 & Vigna unguiculata (L.) Walp. & Fabaceae & Kaaramani \\
\hline 56 & Vigna radiata (L.) R.Wilczek. & Fabaceae & Pachaippayaru \\
\hline 57 & Zea mays L. & Poaceae & Makkasolam \\
\hline
\end{tabular}


International Journal of Trend in Scientific Research and Development (IJTSRD) ISSN: 2456-6470

Table-3: List of ornamentals present in Maruthuvambadi village.

\begin{tabular}{|c|c|c|c|}
\hline S.N & Botanical name & Family & Vernacular name \\
\hline 1 & Bougainvillea glabra Choisy. & Nyctaginaceae & Kaakithapoo \\
\hline 2 & Impaliens balasamine L. & Balsaminaceae & Baalsampoo \\
\hline 3 & Ipomoea quamoclit L. & Convolvulaceae & Mailmaanikkam \\
\hline 4 & Ixora coccinea L. & Rubiaceae & Idlypoo \\
\hline 5 & Mirabilis jalapa L. & Nyctaginaceae & Anthimalli \\
\hline 6 & Pistia stratiotes L. & Araceae & Aagaya thaamarai \\
\hline 7 & Polyalthia longifolia Sonn. & Annonaceae & Nettilinkam \\
\hline 8 & Portulaca grandiflora Hook. & Portulacaceae & Patturoja \\
\hline & Portulaca umbraticola cv. Wildfire & & Buttonrose \\
\hline 10 & Mixed & Portulacaceae & Baadam \\
\hline 11 & Prunus amygdalus Dulcis. & Rosaceae & Roja \\
\hline
\end{tabular}

Table-4: Wild plants present in Maruthuvambadi village.

\begin{tabular}{|c|c|c|c|}
\hline S.N & Botanical name & Family & Vernacular name \\
\hline 1 & Acacia eburnea (L.f.) Willd. & Fabaceae & Karuvelamaram \\
\hline 2 & Acacia farnesiana (L.) Willd. & Fabaceae & Peekaruvelamaram \\
\hline 3 & Acacia pennata (L.) Willd. & Fabaceae & Kaattusigai \\
\hline 4 & Acacia planifrons Wight \& Arn. & Fabaceae & Kudaivelamaram \\
\hline 5 & Albizia amara (Roxb.) B. Boivin. & Fabaceae & Oosillai, Thurinjai, Arappu \\
\hline 6 & Albizia odoratissima (L.f.) Benth. & Fabaceae & Silai vaagai, Karuvagai \\
\hline 7 & Allizzia labbeck(L.) Benth. & Fabaceae & Kaatuvagai maram \\
\hline 8 & Argyreia hookeri C.B.Clarke. & Convolvulaceae & Malaionankodi \\
\hline 9 & Aristida setacea Trin. & Poaceae & Poonthudaipam \\
\hline 10 & Artocarpus integrifolia Lam. & Moraceae & Palaamaram \\
\hline 11 & Asparagus racemosous Willd. & Asparagaceae & Kaatuchedi \\
\hline 12 & Atlantia monophylla Roxb. & Rutaceae & Kaattu elumichai \\
\hline 13 & Azima tetracantha Lam. & Salvadoraceae & Sangillai, Peankuthi \\
\hline 14 & Bambusa bambos (L.) Voss. & Poaceae & Moongil \\
\hline 15 & Bassia latifolia Roxb. & Sapotaceae & Iluppaimaram \\
\hline 16 & Bauhinia tomentosa (L.) Benth. & Fabaceae & Thiruvaachi \\
\hline 17 & Bauhinia variegata (L.) Benth. & Fabaceae & Semmanthaarai \\
\hline 18 & Canthium parviflorum Lam. & Rubiaceae & Kaaraikaai, Sirukaarai \\
\hline 19 & Capparis brevispina Wight \& Arn. & Capparaceae & Aathanthai, Porivilampazham \\
\hline 20 & Carmona retusa (Vahl) Masam. & Boraginaceae & Kuruvipazhamsedi, Kurangu vetrilai \\
\hline 21 & Cassia fistula L. & Fabaceae & Sarakkondrai \\
\hline 22 & Dichrostachys cinerea (L.) Wight \& Arn. & Fabaceae & Vedathalam \\
\hline 23 & Diospyros ferrea (Willd.) Bakh. & Ebenaceae & Irumbilichedi \\
\hline 24 & Lantana camara $\mathrm{L}$. & Verbenaceae & Ounnichedi \\
\hline 25 & Madhuca longifolia (J.Konig) J.F.Macbr. & Sapotaceae & Naattuiluppai \\
\hline 26 & Phoenix loureirii Kunth. & Arecaceae & Eechamaram \\
\hline 27 & Phoenix pusilla Roxb. & Arecaceae & Kaatu eechamaram \\
\hline 28 & Pterocarpus marsupium Roxburgh. & Fabaceae & Veangaimaram \\
\hline 29 & Spondias pinnata (L.f.) Kurz. & Anacardiaceae & Narimangai \\
\hline 30 & Sterculia foetida L. & Malvaceae & Pinarimaram \\
\hline
\end{tabular}




\section{Conclusion}

This survey reveals that plants are still a major source of medicine, food and other purpose for the local communities of most of the portions in our country, because modern healthcare facilities are still insufficient. This simple work may represents a useful and long-lasting document, which can contribute to preserve knowledge on the use of medicinal plants in this village and also stimulate the interest of future generations on traditional healing practices. The information provided in this paper is limited and there is additional scope needed to initiate brief ethnobotanical study. From this survey some important herbals need to be evaluated through phytochemical and pharmacological investigations to discover their potentiality as drugs. This survey is also expected to be useful to botanist, ecologist, crop improvers, plant introducers, students etc. Finally, we concluded that this village was enriched with different kind of plants and furthermore research activities are needed.

\section{References}

1. Anup, Mamun Or Rashid, Shalahuddin and Mamunur "Ethnobotanical Survey of Medicinal Plants Used by Traditional Health Practitioners and Indigenous People in Different Districts of Chittagong Division, Bangladesh". International Journal of Pharmaceutical Science Invention. (2014) PP 1-7.

2. Bhattarai and Madan Kumar "Ethnobotanical Survey of Medicinal Plants from Ilam District, East Nepal". Our Nature. (2016) Vol 14 (1): 7891.

3. Census of India (2011)-Tamil Nadu. District Census Handbook Tiruvannamalai (Village and Town Directory).Directorate of Census Operations Tamil Nadu.Series-34 Part XII-A.

4. Disticraj, S. and Jayaraman, P. "Ethnobotanical Documentation of Few Medicinal Plants in Jawadhu Hills in Tiruvannamalai District of Tamil Nadu". IOSR Journal of Pharmacy and Biological Sciences (2015) Vol 10, Issue 4 PP 08-11.

5. Gbadamosi, I.T. and Egunyomi, A. "Ethnobotanical Survey of Plants Used for the Treatment and Management of Sexually Transmitted Infections in Ibadan, Nigeria". A Journal of Plant, People and Applied Research. (2014) Pane 659-670.
6. Giovannini, P. "Medicinal Plants of The Achuar (Jivaro) of Amazonian Ecuador: Ethnobotanical Survey and Comparison with Other Amazonian Pharmacopoeias". Journal of Ethnopharmacology. (2015) 164 (4), 78-88.

7. Ignacimuthu, S., Ayyanar, M., and Sankarasivaraman, K. "Ethnobotanical Study of Medicinal Plants Used by Paliyar Tribals in Theni District of Tamil Nadu, India". Fitoterapia. (2008) 79,562-568.

8. Johnson, G.M., Nanadagopalan, V. and Doss, A. "Ethnobotanical Survey of Medicinal Plants Used by Traditional Healers in Shobanapuram Village of Pachamalai Hill, Tamilnadu. Adv. Appl. Sci. Res. (2015), 6 (3): 157-164.

9. Lingaiah, M. and Nagaraja Rao, P. "An Ethnobotanical Survey of Medicinal Plants Used by Traditional Healers of Adilabad District, Andhra Pradesh, India, An International Quarterly Journal of Biology \& Life Sciences. (2013) 1 (1): 17-23.

10. Manuel, J., Maciaa, Emilia, G. and Prem, J.V. "An Ethnobotanical Survey of Medicinal Plants Commercialized in the Markets of La Paz and El Alto, Bolivia". Journal of Ethnopharmacology. (2005) 97, 337-350.

11. Ministry of MSME. "Brief Industrial Profile of Tiruvannamalai District". Government of India.www.msmedi-chennai.gov.in/ (2012-2013).

12. Mohammed, R., Israt, J.M., Fahmidul, H.A.K.M., Ariful, H.M., Kanta, P., Rownak, J., Majeedul, H.C., Taufiq, R. "An Ethnobotanical Survey and Pharmacological Evaluation of Medicinal Plants used by the Garo Tribal Community living in Netrakona district, Bangladesh". Advances in Natural and Applied Sciences. (2009) 3 (3): 402418.

13. Omwenga, E.O., Mbugua, P.K. and Okemo, P.O. "Ethno-Medicinal Survey of Important Plants of Samburu Community (Wamba)-Samburu District in Kenya". Journal of Botanical Sciences. (2014) PP 24-29.

14. Pavun Kumar, M. and Suresh Kumar, J. "Ethnobotanical Studies on Thenmudiyanur Village, Tiruvannamalai District, Tamil Nadu, India". International Journal of Plant Sciences, (2011)Vol. 6 Issue 2: 352-357 
15. Poongani, M. and Karpagam, S. 'Ethnobotanical Survey of Medicinal Plants at Vengili, Ambur Taluk, Vellore District, Tamil Nadu, India". World Journal of Pharmacy and Pharmaceutical Sciences. (2017) Vol 6, Issue 02, 779-788.

16. Ramakrishnan, N., Pandian, G., Sampath, K.S. and Hariprasad, P. "Medicinal Plant Diversity in Tiruvannamalai Hill, Tiruvannamalai, Tamil Nadu". Online sharing by Arts College, Tiruvannamalai.

17. Savithramma, N., Sulochana, C.H., Rao, K.N. " Ethnobotanical survey of plants used to treat asthma in Andhra Pradesh, India". Journal of Ethnopharmacology.(2007) 113: 54-61.

18. Shah, Z., Ali, H. and Shariatullah. "Ethnobotanical Survey of Medicinal Plants from Tehsil Dargai, District Malakand, Pakistan".Fuuast j. biol. (2013) 3 (1): 109-113.

19. Thamacin Arulappan M. and John Britto, S. "Some Important Medicinal Plants Used in Gingee Taluk of Villupuram District of Tamil Nadu, India". J. Nat. Prod. Plant Resour. (2014) 4 (3): 13-19.

20. Vijayasankar, R., Perumal, R. and Ravikumar, K. "Plant Resources of Tiruvannamalai District, Tamil Nadu, India". Bishen Singh Mahendra Pal Singh, Dehra Dun, India. (2012). 